\title{
On the Hypothesis of Mitogenetic Radiation.
}

By

\author{
Dr. Nine Choucroun.
}

With 3 Figures in the Text, and Plates VI and VII.

\section{Introduction.}

IN order to explain certain actions influencing at a distance the cellular development of living tissues, various authors have framed the hypothesis of a mitogenetic radiation emanating from cells in the act of division.

Gurwitsch (1) first of all pointed out the mitogenetic influence of cells of embryonic tissues, in the form of an emulsion or otherwise, on the root of the onion or on yeast cells. According to him, this action, which was exerted through sheets of quartz-but not of glass - is due to a radiation, or, to be more explicit, to an ultra-violet radiation of wavelength 2000 Angström units, produced by the active material and capable of regular reflection.

Reiter and Gabor (2), who have since then carried out many more such experiments, also attribute the action to a mitogenetic radiation, the wave-length of which they have measured (according to them nearer to the visible) and can readily follow, so that, for example, they can separate the two rays reflected from the opposite faces of a sheet with parallel faces.

An analogous influence had been observed by M. Joseph Magrou and Mme. M. Magrou by exposing to Bacterium tumefaciens, through a sheet of quartz, a root or a stem of an onion, and later sea-urchin eggs. In the first case they verified the phenomena observed by Gurwitsch, without however affirming the existence of a radiation. In the second case they obtained aberrant larvæ, plutei opaque and with short arms or armless, instead of plutei transparent and elongated as are the normal larvæ. Histological examination seemed to indicate that these differences in appearance were related to differences in structure; in the irradiated larvæ the mesenchymatous cells were more numerous than in the controls. This case also might be explained on the theory of a mitogenetic action.

On the other hand, it did not seem that this action could have been due to a vapour carried by the air, for the controls, placed in the immediate neighbourhood of the lots exposed to radiation, gave a perfectly normal 
development. M. et Mme. Magrou were of the opinion that this action too, just as in the case of the onion root, could be attributed to a hypothetical radiation emanating from the bacteria.

But the action producing malformation was not always evident in the exposed lots, which indeed were not very numerous, and sometimes even the effect was not produced in a single one.

Unsuspected causes of lack of symmetry might conceivably have been at play either in themselves to provoke the observed phenomena or to prevent the action exercised by $B$. tumefaciens from becoming manifest.

It became a necessity, therefore, to establish with certainty the reality of an action causally connected with proximity to the culture. And for that an effort had to be made to realise, in the steps we took, the best possible conditions of symmetry and homogeneity for all the batches, controls and exposed.

\section{Reality of the Phenomena.}

To prove the reality of the phenomena was the task for us, M. Magrou, Mme. Magrou and myself, at the Marine Laboratory of the Institut Océanographique de Monaco (3).

This time we worked with a larger number of batches, about 24, namely, 12 controls and 12 exposed, selected at random. The controls were, for the greater part, placed on a table among the exposed.

Eggs of Paracentrotus lividus, from the very same animal and laid at the same time, were all fertilised in the same vessel by the same supply of sperm. Immediately after fertilisation the eggs were divided in a manner as similar as possible among the small quartz receptacles; these were $3.5 \mathrm{~cm}$. in diameter with optically-worked bottoms. Certain of these receptacles were placed on vessels of the same diameter containing a thick suspension of $B$. tumefaciens in nutritive broth, the suspension coming from a single supply of emulsion.

All the lots, control and exposed, were placed in identical conditions as regards illumination, air supply and temperature, in an incubator kept near $22^{\circ} \mathrm{C}$., which temperature is favourable for the good development of the culture and is borne very well by the eggs used.

Under these conditions we verified the production of abnormalities observed in the earlier work, and the results obtained, based on a number of experiments, allow us to conclude that there is a definite causal connection between the existence of an action and the presence of the culture. But, as before, a certain proportion of the exposed lots (this time always about the same in the successive trials, thanks to the greater number of the receptacles) escaped all action.

It was natural to expect, on account of the degree of homogeneity 
realised, that in each lot a certain proportion of the eggs were more resistant than the others to the injurious action observed, giving rise therefore to larvæ normal in every respect. But it was difficult to admit that entire lots, made up of the same larvæ as the others, could completely escape the action if some cause of lack of symmetry were not in existence.

One might incriminate the culture and ask whether, once it had been subdivided, it retained the same activity throughout. As a fact, in certain vessels, the culture settled out, whereas in others it remained in an emulsion. Sometimes the absence of action coincided with this precipitation of the culture and sometimes it did not do so. In any case, this possible cause of lack of symmetry would disappear if one were to submit all the lots to the action of one single culture, and if in addition this culture were to revolve beneath the batches of seaurchin larvæ kept at rest. This is what I proposed and carried out as follows :-

\section{Realisation of a Homogeneous "Irradiation."}

An annular vessel, A, of pyrex glass, about $30 \mathrm{~cm}$. external and $25 \mathrm{~cm}$. internal diameter, intended to contain the bacterial suspension, rotates around an axis $\mathrm{O}$ (Fig. 1) below another annular vessel, B, which covers

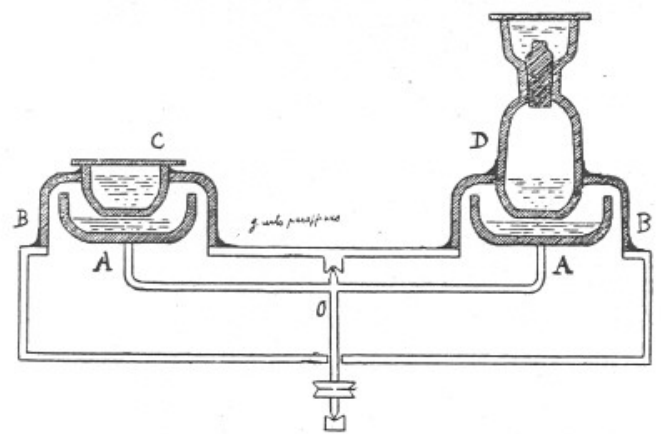

FIG. 1.

it. Vessel B is pierced by circular holes in which are affixed, by joints of paraffin wax, the quartz receptacles, $\mathrm{C}$, which contain the lots of sea-urchin larvæ.

The vessel A is carried by an annular plate of metal which revolves around an axis, $\mathrm{O}$, with a uniform and slow movement, about one turn in eight minutes. The upper vessel rests partly upon the edge of the apparatus, partly upon a central fixed plate, the mechanical disposition of the parts permitting of the complete separation, by means of paraffin wax joints, of the air in contact with the culture from that in contact with the sea-urchin eggs. 
An exactly similar control apparatus functions at the same time, the only difference being that in it the vessel A contains no culture (Fig. 2).

Under these conditions of homogeneous "irradiation" one might expect to observe an absolute identity in the action. Now, in the experiments carried out at the Marine Laboratory of Banyuls, we indeed observed progress in this respect; but in certain experiments, irregularities existed (exposed lots remaining normal) which it became difficult to explain on the hypothesis of a radiation once homogeneity of irradiation had been realised.

On the other hand, the photographic plate substituted for the seaurchin eggs never revealed the supposed radiation, in spite of very long exposures (48 hours). Stated more precisely, with the conditions

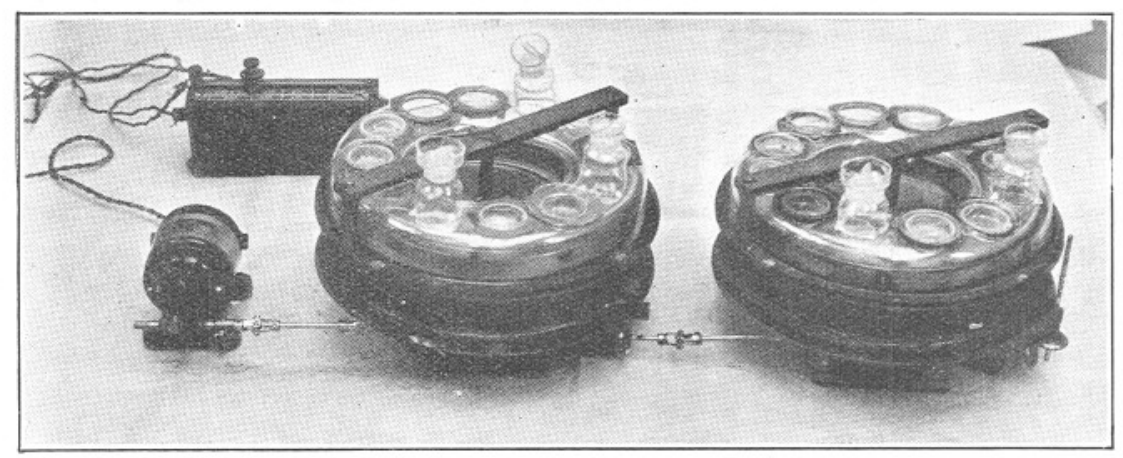

FIG. 2.

under which the phenomena became manifest in the eggs, the plate, even when sensitised to the extreme ultra-violet, received absolutely no impression. I must say that for many physicists this negative result sufficed to render doubtful the existence, in this case, of a mitogenetic radiation.

I then applied myself, in a new piece of work carried out this time at the Marine Laboratory at Plymouth, to test this hypothesis of radiation.

\section{It is Not a Radiation which Acts.}

To investigate the above proposition, certain of the quartz capsules were replaced by quartz flasks, D, with ground stoppers, the base of which was intentionally constituted of capsules already used in the preceding experiments, and of which the stoppers were surrounded by a trough filled with frequent changes of sea-water (Fig. 1).

Each experiment consisted of twelve lots exposed (8 open capsules and 
4 stoppered flasks) carried by the apparatus containing B. tumefaciens and 12 controls ( 10 open capsules and 2 stoppered flasks) carried by the other apparatus. According to the radiation hypothesis, the action should be shown in the same manner in the stoppered flasks and in the capsules. One should observe in these flasks an abnormal development simultaneously with a normal development in the control flasks of the blank apparatus.

Now, never did I observe any abnormality in the exposed stoppered flasks. The same culture of $B$. tumefaciens which exercised a strong action on the eggs in the capsules produced no effect on the eggs in the stoppered flasks; the water-seal sufficed to stop all action. In three of the experiments, all the capsules exposed gave abnormal developments, whilst the four stoppered flasks gave normal developments as well as the twelve receptacles (capsules or flasks) of the blank apparatus.

In the photograph reproduced here by way of example, it may be seen that in the lots exposed in stoppered flasks the development is normal and comparable to what it is in the control vessels, whereas the anomalies are striking in the lots of the exposed capsules (Plate VI).

No longer, then, can one explain the observed action at a distance by a special radiation which exerts its effect through comparable media and would clearly reach equally well both the batches of eggs in the capsules and in the stoppered flasks.

More generally, and this is obvious, it is not possible to explain the observed phenomena by any action exerted through quartz vessels, for then it could not possibly suffice to close the flasks to stop all action.

\section{Detailed Description of an Experiment.}

Before speaking of the observations, that I have been able to carry out, in the light of a different interpretation, I should like to describe in detail one experiment, laying emphasis upon the precautions that I had to take to realise, in the steps we took, the greatest possible similarity between the exposed lots and the controls.

All the receptacles were washed for a long time with concentrated hydrochloric acid, then with water, then with alcohol, then with distilled water, after which they were sterilised. The same precautions were taken for the vessel, A, before containing the culture, for the perforated covers, $\mathrm{B}$, also for the non-perforated covers, $\mathrm{B}_{1}$, which were to protect the vessel A during filling or transfer to the mechanical apparatus (Fig. 3). The receptacles, capsules and flasks, were divided, haphazard, between the two perforated covers resting on sterilised paper. All the joints were sealed with paraffin wax (M.P. $50^{\circ}$ C.), then with soft paraffin (M.P. $38^{\circ}$ C.) to fill up possible cracks. 
The sea-urchin eggs, of the species Echinus miliaris, coming from one oviposition were washed for a long while with fresh sea-water, then fertilised all at once by the same sperm suspension; they were then separated by decantation from the water in which they had been fertilised and finally suspended in a volume of sea-water sufficient for the eggs to develop well and withal small enough for their concentration in each receptacle to render observation easy. They were then divided uniformly, by the aid of a sterilised glass spoon, between the various receptacles, ladling out alternately the lots for the apparatus with $B$. tumefaciens and those for the control.

Once more must I insist upon the necessity for respecting the conditions of symmetry up to a point which may appear puerile.

The Bacterium tumefaciens was cultivated on agar (at a temperature of $38^{\circ}$ C.) in large Roux flasks. It was then emulsified in ordinary

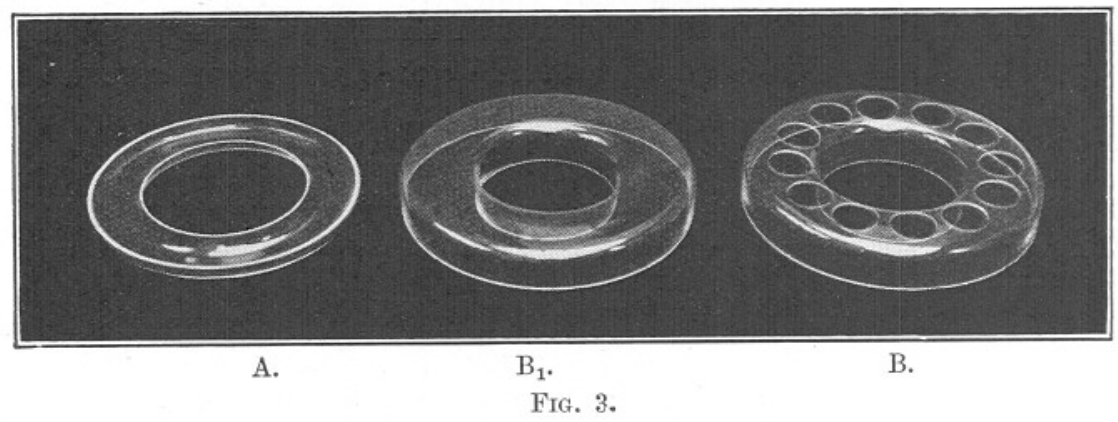

broth $\mathrm{Ph} .7 \cdot 4$ and the emulsion from each flask was poured with aseptic precautions into the vessel $A$ furnished with its cover, $B_{1}$. With three Roux flasks a thickness of about $5 \mathrm{~mm}$. emulsion was obtained. This vessel A and the empty control vessel, both furnished with their protective covers, $B_{1}$, were carried on the rotating plates of the apparatus. For these covers, $B_{1}$, were substituted the perforated covers $B$, carrying the vessels the bases of which were then within a few millimetres (about eight) of the free surface of the culture. The inner and outer edges of these covers were paraffined with a brush and the motors turning the plates bearing the vessels A were started. The experiment was allowed to proceed till the characteristic " pluteus " stage was reached, renewing, about every four hours, the sea-water contained in the troughs of the stoppered flasks so as to maintain a fresh water-seal, free from impurities.

The first experiment was carried out at a temperature of $17^{\circ} \mathrm{C}$., the apparatus being enclosed in a water-oven regulated for this temperature. But the renewing of the water of the water-seal was difficult inside the oven. Accordingly in the following experiments the apparatus were 
simply placed upon the laboratory table. If necessary the general temperature was lowered so as not to exceed $20^{\circ} \mathrm{C}$. Moreover, some additional control lots were placed right up against the apparatus containing $B$. tumefaciens as a test that, as was already known, the abnormalities were not produced by the very small temperature differences which might exist between the atmospheres of each apparatus.

As regards observation, each lot was examined in its position by the aid of a binocular glass which allowed one to have in the field, and in its depth, a sufficiently great number of larvæ for an estimate to be made of the relative proportion of the normal and abnormal larvæ in each lot.

All the lots having been examined and the observations recorded; photographs of the experiment were made, working in such a manner as to be entirely ignorant as to whether a control or an exposed lot was being taken. From each lot, after shaking, a small quantity of the emulsion was taken and placed in a numbered test-tube. To each testtube were added a few drops of $1 \%$ chloral to stop the movement of the larvæ which then became aggregated at the bottom of the tube. Using a very finely drawn-out pipette a small quantity of liquid containing larvæ was removed from each tube and placed upon a microscope slide. The number of the corresponding lot was written on a label on the slide and hidden.

Then from among all the possible fields a choice was made of one which gave the best representation of the general aspect of the preparation.

It is only thanks to these precautions, of which some might seem puerile, that the photographs taken represent objectively the general aspect of the lots examined. This was indispensable to allow those who only saw the photographs to form an independent opinion.

\section{Attempt at Explanation.}

Let us now return to the phenomenon itself.

If it seems no longer possible to explain the anomalies observed by a radiation, nor in a more general form by any action involving "passage through," it is equally impossible to doubt the existence of an action clearly established and confirmed by so many new experiments.

It seems difficult then to avoid thinking that something material, given off by the culture, reaches-in spite of the paraffin wax jointsthe medium in which the eggs are developing, travelling round the wall of the receptacles.

It is fairly frequently observed that a bubble develops at the base of the 
exposed capsules. One may then raise the question as to whether a film, monomolecular perhaps, may not start from these, spread over the wall, insinuate itself between this wall and the paraffin joint, and introduce into the medium, in which the eggs are developing, very minute amounts of a substance which suffice however-and this is very remarkable - to exercise on the eggs an exceedingly injurious effect.

Concerning this I have made the following observations: in two experiments, certain quartz capsules contained only sea-water both on the apparatus with $B$. tumefaciens and on the blank apparatus. On each perforated lid there were five capsules with eggs, five with sea-water only and two stoppered flasks with eggs.

After having ascertained that the bacterial culture had acted on the lots of sea-urchins exposed in the capsules (never any action on those in the flasks) I withdrew the active culture; then I placed sea-urchin eggs (coming from a newly fertilised batch) in the sea-water which had been exposed, and in the unexposed sea-water of the blank apparatus.

Development was very abnormal in the receptacles which had been exposed to $B$. tumefaciens and normal in the controls. In one of the two experiments, stopped at the gastrula stage so as to be able to photograph certain peculiarities, the injurious action had been particularly intense. In one of the lots (Plate VII) the eggs had not even divided; in the others many eggs had not reached the blastula stage. In the majority of the lots advanced cytolysis could be observed even before the gastrula stage had been reached. This would seem to indicate that the active impurity is specially active during the first stages of development. According to our hypothesis of the transport of something material, however small it be, by a monomolecular film, it is understood that exposed sea-water must be, for the eggs introduced into it, a different medium from that in which the eggs are when themselves directly exposed to the culture. In this case, the active impurity, nothing at the start, goes on increasing during the experiment; whereas in the former case there has been, right from the beginning, a relatively great concentration.

Thus, exposed sea-water has been modified thanks to proximity to the culture, and this in spite of precautions which seemed adequate to guard against all material action.

The objection has been raised that the sea-water itself might very well have been modified by a hypothetical radiation. But then it would be impossible to understand why this modification had not been produced in the sea-water of the stoppered flasks containing eggs, necessitating an abnormal development for the eggs. 


\section{SUMMARY.}

1. The observed "action at a distance" undoubtedly exists.

2. This action cannot be ascribed to a radiation, nor more generally to an action "through the wall" of the receptacles.

3. This action must then be exercised "across the surface" of the wall of the receptacles by a mechanism which remains to be explained. This seems, however, to be of great interest in view of the apparently very small mass of the substance concerned in the action.

4. The action of something material is rendered probable from the fact that the eggs develop very abnormally in sea-water previously exposed to the active culture.

5. Although these experiments have only been carried out upon sea-urchin eggs, their conclusions may be valid in the case of the very numerous experiments concerning mitogenetic radiation.

In the first experiments it was thought that the sea-urchin eggs had been shielded from the action of any material emanating from the culture. An additional precaution (stoppered flasks) sufficed, however, to bring it about that the better isolated eggs remained insensible to the action at a distance which continued, however, to be exercised upon the other receptacles, which were less efficiently protected.

Experiment has shown that a water-seal sufficed to protect the eggs against all action producing deformity. If in this case abnormalities had again been found it would have been necessary to substitute sealed flasks for stoppered flasks. Only then, and if the abnormalities had again been found in sealed flasks exposed, but not in sealed control flasks, would it have been possible to conclude that the existence had been established of an action at a distance by means of a radiation, or more generally, an action exercised through quartz.

As far as the actions not studied here are concerned the conclusion must certainly be drawn that the existence of a radiation has not been proved.

It would at least be necessary, in order to carry conviction in this respect, that rigorous precautions should be taken to hinder absolutely all transport of material between the activating substance and that used as a test in the observations.

It has been possible to carry out these researches thanks to a grant from the Institut de Biologie physico-chimique, founded by M. Edmond de Rothschild. 
On the other hand, I must specially thank Dr. E. J. Allen for the hospitality afforded to me in his laboratory. It has been very valuable to me, in the course of my work, to profit by the resources of the laboratory and above all from the friendly atmosphere created by Dr. Allen and the numerous research workers surrounding him. I should like to thank them all.

\section{REFERENCES.}

1. Gurwitsch, A. Compt. rend. Acad. Sci., Paris, 1927, 184, pp. 841 and 903 .

2. Reiter, T. and Gabor, D. Zelltheilung und Strahlung. J. Springer, Berlin, 1928.

3. Magrou, J., Magrou, M., and Choucroun, N. Compt. rend. Acad. Sci., Paris, 1929, 188, p. 733.

\section{EXPLANATION OF PLATES.}

\section{PLATE VI.}

Experiment III. Shows normal development of sea-urchin larvæ in stoppered flasks and control vessels, and abnormal development in exposed quartz capsules.

\section{PLATE VII.}

Experiment VIII. Shows abnormal development of sea-urchin eggs which had been placed in "exposed" sea-water, and normal development of controls. 


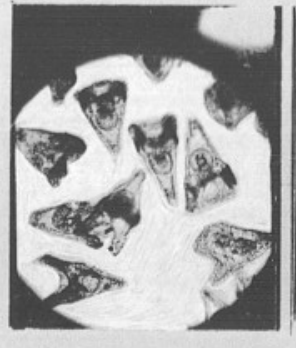

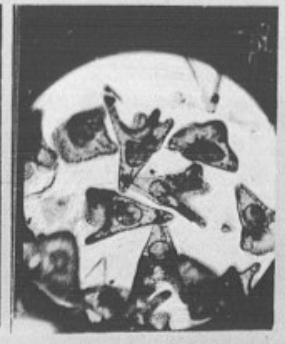

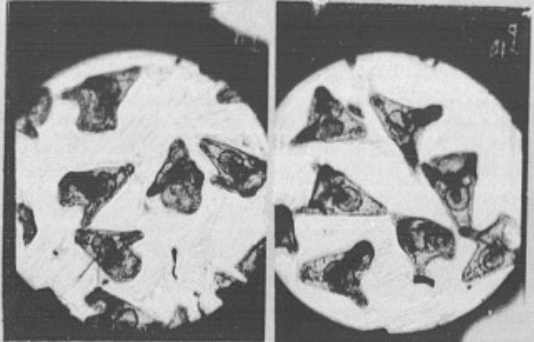

flacons bouchés émeri "trautés"

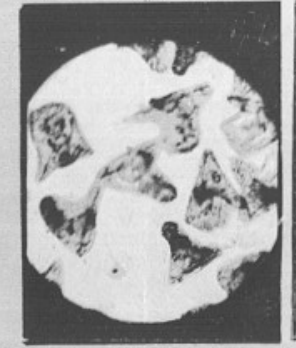

Gémoius
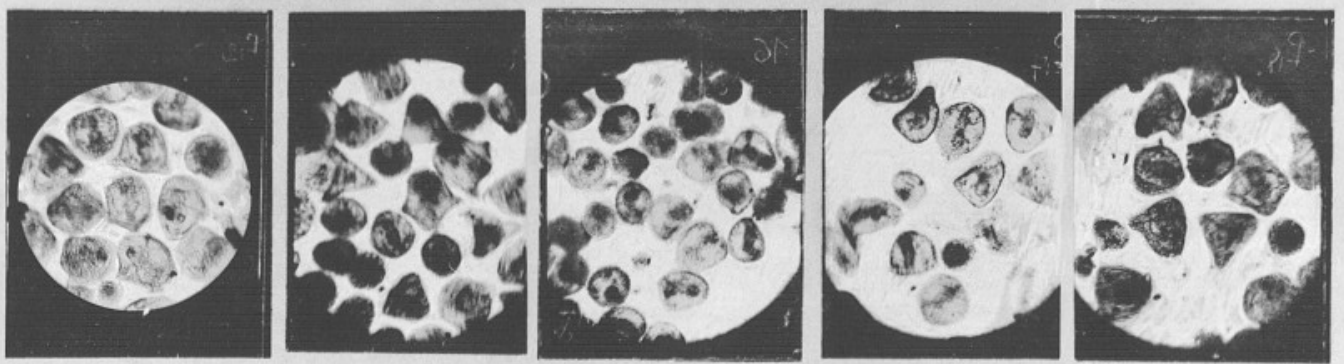

capsules quartz "traités" 


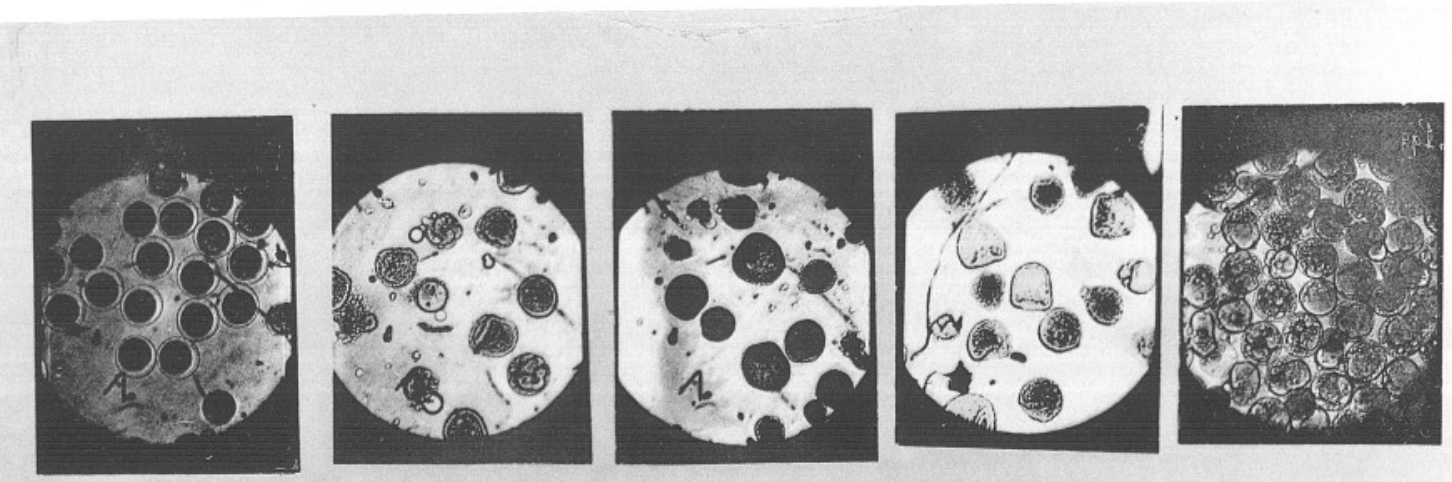

ceufs dans eau de mer "exposée"

ceup daus

eau do mer

témoin

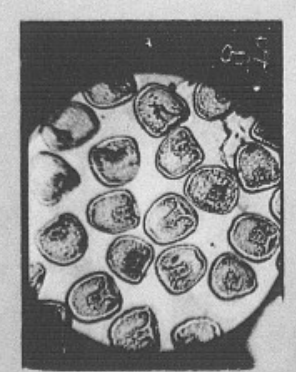


